\title{
UNIPOTENT CONJUGACY IN GENERAL LINEAR GROUPS
}

\author{
J. L. Alperin
}

Mathematics Department, University of Chicago, Chicago, Illinois, USA

Let $U(n, q)$ be the group of upper uni-triangular matrices in $G L(n, q)$, the $n$ dimensional general linear group over the field of $q$ elements. The number of $U(n, q)$ conjugacy classes in $G L(n, q)$ is, as a function of $q$, for fixed $n$, a polynomial in $q$ with integral coefficients.

Key Words: Unipotent conjugacy.

Mathematics Subject Classification: Primary 20C15; Secondary 20 D06.

A conjecture for at least forty years states that the number of conjugacy classes in $U(n, q)$, the group of upper uni-triangular matrices over a field with $q$ elements, is, as function of $q$, with $n$ fixed, given by an integral polyonomial in $q$ (e.g., see Isaacs, 1995; Robinson, 1998). We show here that a similar result is easily established.

Theorem. The number of $U(n, q)$-conjugacy classes in $G L(n, q)$ is, as a function of $q$, for fixed $n$, given by a polynomial in $q$ with integral coefficients.

Hence, it is easy to count the orbits of $G L(n, q)$ under the conjugation action of $U(n, q)$ but seems hard to do the same for the group $U(n, q)$ itself! We fix some further notation. Let $V(n, q)$ be the vector space of column vectors, a module for $G L(n, q)$. Recall that a flag is a totally ordered set of $n-1$ nonzero proper subspaces of $V(n, q)$. For $g$ in $G L(n, q)$ let $f(g)$ be the number of flags fixed by $g$, so it is zero unless $g$ is conjugate with an element of $B(n, q)$ the group of upper triangular matrices. For a unipotent element $u$ of $G L(n, q)$ let $\pi(u)$ be the partition of $n$ given by the Jordan form of $u$.

Lemma 1. If $K$ is a unipotent conjugacy class of $G L(n, q)$ with representative $k$ then

$$
|K \cap U(n, q)|=\frac{|K|}{|G L(n, q): B(n, q)|} \cdot f(k)
$$

Consider the set of all pairs $(x, W)$ where $x$ is in $K, W$ is a conjugate of $U(n, q)$ in $G L(n, q)$, and $x$ lies in $W$. The cardinality is equal to the product of the number

Received November 2, 2004; Revised January 28, 2005. Communicated by A. Turull.

Address correspondence to J. L. Alperin, Mathematics Department, University of Chicago, 5734 University Avenue, Chicago, IL 60637, USA; Fax: (773)702-9787; E-mail: alperin@math.uchicago.edu 
of conjugates of $U(n, q)$, i.e., $|G L(n, q)| /|B(n, q)|$, with $|K \cap U(n, q)|$. However, it is also equal to the product $|K| \cdot f(k)$.

Lemma 2. For a fixed partition $\pi$ of $n$, the value $f(x)$ for $x$ a unipotent element with $\pi(x)=\pi$, as a function of $q$, is given by a polynomial in $q$ with integral coefficients.

Since this number is simply the value at $x$ of the permutation character of $G L(n, q)$ on the cosets of $B(n, q)$, basic facts about unipotent characters imply the result immediately (Green, 1955). However, it is completely elementary to see directly. Working by induction, one can assume the result holds when $n$ is replaced by $n-1$. Say $\pi=\left(\lambda_{1}^{a_{1}}, \lambda_{2}^{a_{2}}, \ldots, \lambda_{r}^{a_{r}}\right)$, where the $\lambda_{j}$ are a decreasing sequence of positive integers. The number of one-dimensional subspaces of $V(n, q)$ left invariant by $x$ such that the partition of the action of $x$ on the quotient space reduce one $\lambda_{1}$ by one, is $\left(q^{a_{1}}-1\right) /(q-1)$, the number of one-dimensional subspaces left invariant by $x$ such that the partition of the action of $x$ on the quotient space reduce one $\lambda_{2}$ by one is $\left(q^{a_{2}+a_{1}}-q^{a_{1}}\right) /(q-1)$, and so on, so the result follows.

We can now prove the theorem. The desired number, by the Burnside lemma, equals, where $G=G L(n, q)$,

$$
\frac{1}{|U(n, q)|} \sum_{u \in U(n, q)}\left|C_{G}(u)\right|=\frac{1}{|U(n, q)|} \sum_{K}|K \cap U(n, q)|\left|C_{G}(k)\right|,
$$

where $K$ runs over the unipotent conjugacy classes, and $k$ is a representative of $K$. But this is then, by Lemma 1 , equal to

$$
\sum_{K} \frac{|K|}{|U(n, q)|} \cdot \frac{|B(n, q)|}{|G L(n, q)|} \cdot \frac{\mid(G L(n, q) \mid}{|K|} \cdot f(k)=(q-1)^{n} \sum_{K} f(k),
$$

which is what is needed by Lemma 2 .

The above proof, aside from being very short, is quite formal. This means that it applies to other Lie type groups as well. We shall, however, discuss below another direction for extensions, in fact to the question that originally arose.

First, however, it is interesting to contemplate the very unlikely possibility of a proof of the original conjecture on $U(n, q)$ by descent from the theorem proven, perhaps by considering each of the double cosets of $U(n, q)$ in $G L(n, q)$. Certainly, the double coset that seems the most complicated is the subgroup $U(n, q)$ itself. It is also hard to imagine that the original conjecture is false but the sum of the counts over the double cosets is still polynomial, as we know it is. One possible advantage of a proof by descent is that it would use the fact that $U(n, q)$ is a subgroup of $G L(n, q)$ and not just the usual generators and relations of $U(n, q)$. Could there be very delicate deformations of $U(n, q)$ which do not satisfy the original conjecture? This would be quite relevant.

Finally, we note that the theorem also holds for $B(n, q)$-conjugacy as well, directly following the proof above. The final formula, for the number of $B(n, q)$ conjugacy classes in $G L(n, q)$, is simply the sum, over representatives $x$ of each of the conjugacy classes of $G L(n, q)$ which intersect $B(n, q)$ of the value $f(x)$, so what we require is a version of Lemma 2 for such a sum. For such an element $x$, let $\tau(x)$ be the partition of $n$ given by the distribution of the eigenvalues of $x$, so each part 
of the partition is the multiplicity of one of the eigenvalues. Thus, for example, if all the eigenvalues are equal, then $\tau(x)=1^{n}$. In addition, there is associated with each part of $\tau(x)$, a further partition given by the Jordan form. The number of conjugacy classes $x$ with $\tau(x)$ and the other associated partitions fixed is then a polynomial in $q$, so it only remains to show that the value $f(x)$ is a polynomial in $q$ only depending on $\tau(x)$ and these further partitions. But any one-dimensional subspace of $V(n, q)$ left invariant by $x$ is in one of the generalized eigenspaces of $x$, so the count of invariant one-dimensional subspaces is the sum over the eigenvalues of a calculation that we have essentially done in the course of the proof of Lemma 2. Hence, our main result does hold for $B(n, q)$ also. It would be interesting to know that it also holds for parabolic subgroups and their unipotent radicals, as seems quite likely.

\section{REFERENCES}

Green, J. A. (1955). The characters of the finite general linear groups. Trans. Amer. Math. Soc. 80:402-47.

Isaacs, I. M. (1995). Characters of groups associated with finite algebras. J. Algebra 177(3):708-730.

Robinson, G. R. (1998). Counting conjugacy classes of unitriangular groups associated to finite-dimensional algebras. J. Group Theory 1(3):271-4. 